\title{
Incidence of spasm at the site of previous successful transluminal coronary angioplasty: effect of ergometrine maleate in consecutive patients
}

Sir,

Quyyumi et al (1986;56:27-32) report an increased sensitivity to ergometrine maleate (ergonovine maleate) at the site of previous successful transluminal coronary angioplasty in over a third of their patients. This finding has obvious clinical implications, especially with regard to restenosis. Unfortunately the illustrations of the coronary angiograms in fig 4 leave much to be desired. Fig $4 \mathrm{~b}$ which is meant to show "successful dilatation of the stenosis" seems to show a rather severe narrowing in the proximal left anterior descending artery which does not appear to be much different from the original lesion shown in fig 4a and which was the very same site where spasm resulted from intravenous ergometrine shown in fig 4c. Thus I wonder if one is justified in calling the response an increased sensitivity if the coronary arterial lesion before ergometrine was already very tight. Although single frames from cineangiograms often do not do justice in depicting coronary lesions, I think the examples in fig 4 are certainly not very convincing. Authors generally tend to show their best products in their presentation; I wonder if Quyyumi et al have better examples than those published.

\section{Tsung $\mathrm{O}$ Cheng,}

Department of Medicine,

The George Washington University Medical

Center,

The HB Burns Memorial Building,

2150 Pennsylvania Avenue, NW,

Washington, DC 20037,

USA.

This letter was shown to the authors, who reply as follows:

Sir,

We thank Dr Cheng for his comments. As Dr Cheng rightly points out, single frames from cineangiograms often do not do justice in depicting lesions. We have therefore reproduced in biplane views (figs 1 and 2 ) the pre-angioplasty and postangioplasty lesion from the same patient as in our paper. We are sure that there can be little doubt that this patient did have a successful angioplasty.

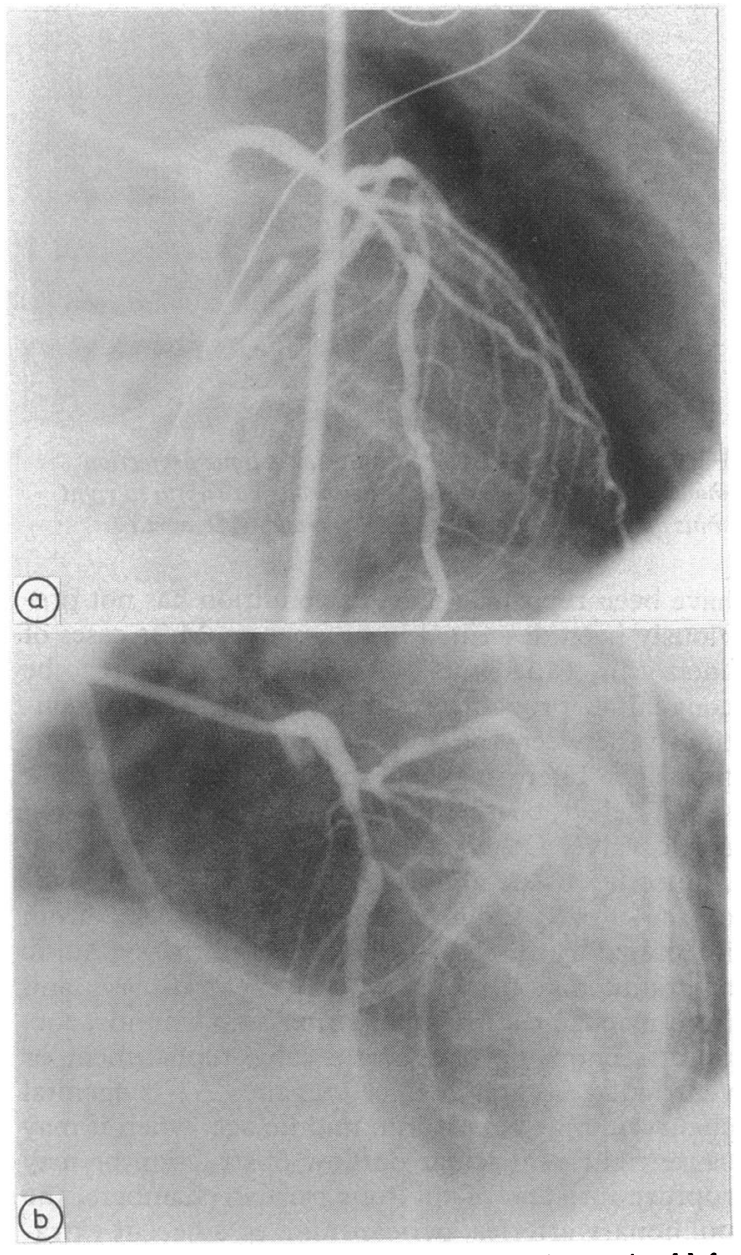

Fig 1 Cineangiograms of the original lesion in proximal left anterior descending coronary artery. (a) Anteroposterior view; (b) left anterior oblique cranial view.

\section{LM Shapiro,}

M Raphael,

A Rickards,

K Fox,

National Heart Hospital,

Westmoreland Street,

London W1M 8BA. 

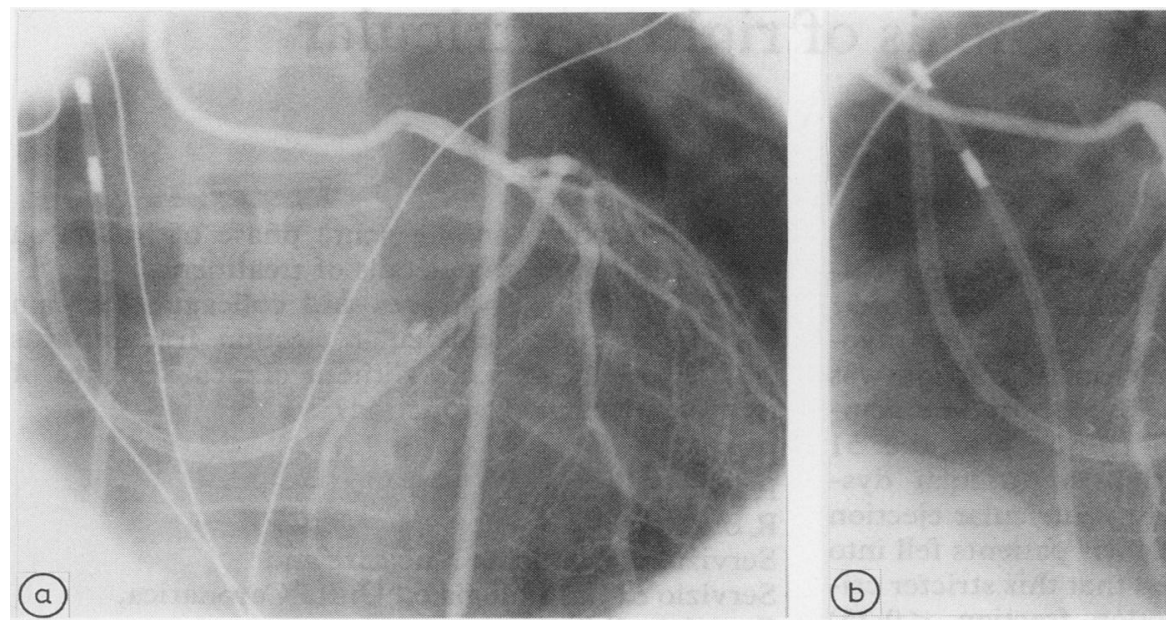

Fig 2 Cineangiograms of similar views of the left anterior descending coronary artery demonstrating successful dilatation of the stenosis. (a) Anteroposterior view; (b) left anterior oblique cranial view.

\section{Early results after percutaneous transluminal coronary angioplasty in 400 patients}

Sir,

Dr Sowton and coworkers $(1986 ; 56: 115-20)$ report five angioplasty related deaths in the first 400 patients who underwent percutaneous transluminal coronary angioplasty at Guy's Hospital. In 1985, one of the authors of this study, Dr Timmis, presented the preliminary results to the Junior Cardiac Club. At that time, 254 patients had undergone the procedure and there had been six deaths. The mortality figures are recorded in the minutes of the Club, which are not published or distributed but are held by the President of the Club and available for inspection by members.

I should be grateful if the authors would explain whether changes in protocol or inclusion criteria account for these discrepancies in mortality figures.

Peter Wilmshurst, Department of Cardiology, St Thomas's Hospital, London SE1 7EH.
This letter was shown to the authors, who reply as follows:

Sir,

The results in our paper were for the first angioplasty, as stated in our introduction. The presentation to the Junior Cardiac Club by $\mathrm{Dr}$ Timmis included results and complications in second or third angioplasties of patients who had recurrent angina. As also stated in our paper the results in patients who have relapse over a five year period will be presented in a separate communication.

Adam D Timmis,

Edgar Sowton,

Department of Cardiology,

Guy's Hospital,

St Thomas Street,

London SE1 9RT. 